\title{
A checkup for the flu vaccine
}

\author{
Influenza causes almost 650,000 deaths worldwide each year, yet a long-lasting, protective vaccine remains elusive. \\ Global investment—both scientific and financial-in a universal flu vaccine is overdue.
}

- ( ach year in February and September, the World Health Organization (WHO) issues its recommendations for the virus composition of the seasonal influenza vaccine for the Northern and Southern hemispheres, respectively. The recommendations are based on global surveillance and clinical data on circulating virus strains and are issued 6-8 months prior to the start of flu season in each hemisphere to enable manufacture and distribution of the vaccines. Yet despite annual updates, vaccine efficacy ranges from only $10 \%$ to $60 \%$, with this year's vaccine for the Northern hemisphere estimated to be $36 \%$ effective in the United States (http://www.cdc.gov/flu/professionals/vaccination/effectiveness-studies. $\mathrm{htm} /$ ). Improvements to the flu vaccine are clearly needed.

The low efficacy of seasonal flu vaccines is due to high antigenic diversity. Human infections are primarily caused by two types of influenza virus, types A and B, and there are dozens of strains of each type. Formulations of seasonal flu vaccines include inactivated versions of two type A viruses and one type $B$ virus (in trivalent vaccines) or two of both type $A$ and $B$ viruses (in the newer quadrivalent vaccines). If the virus strains chosen six months ahead do not match the predominant circulating strains during a flu season, the ability of the vaccine to protect against infection is diminished.

Incorrect predictions are not the only cause of reduced vaccine efficacy, however. Influenza A and B viruses undergo frequent genetic variation through antigenic drift, causing small changes that enable them to evade antibody responses elicited by a closely related vaccine virus. Influenza A also undergoes antigenic shift, in which viral strains-including those that infect different species-can reassort their genetic material to generate a new virus with completely distinct phenotypic and immunogenic properties. Both antigenic drift and shift can contribute to vaccine failure and influenza epidemics, and antigenic shift in influenza $\mathrm{A}$ is associated with flu pandemics, most recently in 2009.

Influenza vaccine manufacturing-most commonly using chicken eggs-also creates conditions that can reduce effectiveness. Viruses propagated in eggs can acquire mutations that make them antigenically distinct from circulating viruses, resulting in poor antibody recognition of epidemic virus strains.

A universal influenza vaccine that provides broad and lasting protection against multiple virus strains would ideally stay ahead of the viral antigenic changes, increase protection against respiratory illness, and reduce the need for annual immunization. To this end, US Senators proposed a new bill in February, the Flu Vaccine Act, requesting \$1 billion over 5 years for the country's National Institutes of Health (NIH) to fund efforts to develop a universal flu vaccine. This investment would substantially boost NIH funding of influenza research, estimated at \$215 million for 2018, down from $\$ 304$ million in 2013. Funding of a universal flu vaccine is lower still-last year, the US National Institute of Allergy and Infectious Diseases (NIAID) spent only $\$ 64$ million on a universal flu vaccine. Compared to $\$ 10.4$ billion in annual influenza-related medical costs and $\$ 16.3$ billion in lost revenue in the United States alone, a \$1 billion investment seems well worth the expense (http://www.cdcfoundation.org/businesspulse/fluprevention-infographic/). More recently in March, the NIAID published a strategic research plan focused on influenza transmission and pathogenesis, immunity, and vaccine design as three key components in the rational development of a universal flu vaccine (J. Infect. Dis. doi:10.1093/infdis/ jiy103, 2018).

A more comprehensive understanding of influenza pathogenesis may improve the rate of success of universal vaccine designs. Seasonal flu vaccines generate an immune response narrowly focused on the globular 'head' of the viral hemagglutinin (HA) protein, which sticks out from the viral envelope. Several efforts to develop a universal vaccine are targeting different viral antigens or epitopes to broaden the immune response beyond the HA head.

For instance, NIAID has initiated a clinical trial testing the safety and immunogenicity of vaccination with a ferritin nanoparticle containing the HA protein either alone, or in combination with a DNA plasmid encoding HA. In animals, the nanoparticle elicited antibodies to both the HA 'stem' and head that neutralized antigenically distinct viruses, an effect that may be attributed to the HA-stem-specific antibodies.

Taking an alternate approach to targeting HA, Vaccitech in the United Kingdom is testing in phase 2 trials a modified vaccinia virus Ankara vector encoding influenza nucleoprotein and matrix-1 (M1) protein. Their vaccine induced $\mathrm{T}$ cell responses and reduced the intensity of symptoms and the duration of virus shedding - which enables viral spread-in humans. This T cell-focused approach is in sharp contrast to the majority of influenza vaccine designs that measure neutralizing antibody as reflective of protective efficacy. And Vaccitech is not alone in trying to engage T cells: BiondVax's M-001 vaccine similarly elicits $\mathrm{T}$ cell responses to conserved viral epitopes from influenza $A$ and $B$ strains and is in trials as a standalone vaccine and as a priming step before administration of the seasonal flu vaccine.

To induce immune responses beyond one or two viral proteins, FluGen, a Wisconsin-based company, has developed a live, attenuated virus that lacks the M2 protein but makes all of the other viral proteins after infection of cells. Delivered as a nasal spray, it will undergo human testing in May in Europe.

While these vaccines are all designed to protect against more than a single influenza strain, whether they are 'universal' vaccines is unknown, and some feel we are still a long way from that goal. Vaccitech CEO Tom Evans estimated that their vaccine, which is in advanced clinical development-if successful-would take 5-7 years to reach market. And even then, it is not clear that existing manufacturing capacity is sufficient to meet the global demand for an entirely new vaccine modality. Investments are therefore needed across the board-in basic research and in vaccine development, but also in manufacturing technologies and infrastructure to respond more rapidly in the advent of pandemics and in the absence of a universal flu vaccine. Without sustained commitment, we will always be a season behind. 